\title{
Aerosol particles characteristic of plant leaves
}

\author{
Fu Hai-ming \\ College of Environmental Science and Engineering, State Environmental Protection Engineering \\ Center for Pollution Treatment and Control in Textile Industry, Donghua University,Shanghai \\ 201620,China, E-mail: fhm@dhu.edu.cn.
}

Keywords: Particulate matter PM; Bio-aerosols; particles diameter distribution; tree leaves

\begin{abstract}
Particles emitted from vehicles are part of the major sources of air pollution in urban areas. It causes the incidences increasing of allergies, allergic diseases and asthma and climate change which is likely to impact plants and animals, as well as microorganisms. Particles mass measurements were used to assess primary biological aerosol particles concentration. Samples from tree were collected and analyzed as the purpose of this paper. The particles diameter distribution existing in the leaves is examined to evaluate the potential bio-aerosol concentrations. In this study, we focus on components of the particles, toxicology of particles via selecting four kinds of trees leaves analysis. Experiments show that fungal spores to be the most important contributors and the distribution of the particles diameter in the leaves is Poisson distribution, 0-1 micro-meter particles are about 5\%-8\% of total aerosol particles. The experiment reveals that leaves are affected directly by deposition of atmospheric pollutants and deposition particles interference plants absorb sunlight and carbon dioxide as well as the process of release oxygen and waters and affecting the health and growth of plants, at same time the tree leaves can reduce the concentration of particulate matter in the air, and reduce the daily mortality of population.
\end{abstract}

\section{Introduction}

The incidence of asthma and allergic disease was increasing in the world [1,2]. Increasing exposure to allergens was one consequence of global climate change. As temperatures increase plant distribution changes in latitude and elevation, and the growing season lengthens in which airborne pollen was produced [3, 4]. Increased concentrations of carbon dioxide can produce increased rates of pollen production [5]. Areas that experience increased humidity can also have an increase in airborne mold spores [6]. Airborne bio aerosols plays an important role in atmospheric environment, they are a major component for evaluating total long-term personal exposures to both PM10 $(<10 \mu \mathrm{m}$ in aerodynamic diameter $)$ and PM2.5 fraction $(<2.5 \mu \mathrm{m}$ in aerodynamic diameter $)$ particulate matter (PM)[7]. Exposure to both ambient and indoor PM has been the focus of intense studies.

The smallest particles are viruses $(0.005 \mu \mathrm{m}<\mathrm{r}<0.25 \mu \mathrm{m})$ The larger particles include bacteria $(\mathrm{r}>\sim 0.2 \mu \mathrm{m})$, protozoa $(\mathrm{r}>2 \mu \mathrm{m})$, algae and fungi $(\mathrm{r}>0.5 \mu \mathrm{m})$, pollen grains $(\mathrm{r}>5 \mu \mathrm{m})$, plant debris like leaf litter, parts of insects and human and animal epithelial cells (supposed $r>$ $1 \mu \mathrm{m})$.Bio-aerosols or particulate matter (PM) which is biological in origin are predominantly comprised of plant pollen and microorganisms (mold and bacteria) or microbial metabolites [9] and can induce allergic, toxic, and infectious responses in exposed individuals.

Symptoms of exposed individuals include coughing, wheezing, runny nose, irritated eyes or throat, skin rash, diarrhea, aggravation of asthma, headache, and fatigue. Immunological reactions can include asthma, allergic rhinitis [10]. A survey of literature indicated that exposure to bio-aerosol can result in adverse health effects in a large percentage of the population [11]. Data suggests that biological sources of PM account for between 5\% and $10 \%$ of the urban and rural 
aerosol composition [12]. Particulate matter of biological origin has been shown by Salvaggio and Aukrust (1981) to be made up of fungi, bacteria, plant pollen, and spore material, all of which have been associated with allergic symptoms. Although pollen is widely studied as an aeroallergen, comparably little knows about ambient concentrations of fungal spores. Salvaggio and Aukrust (1981) indicated cladosporium spores outnumbered pollen spores in the ambient air at a ratio of approximately 1000 to one.

Bacteria and fungi are important components of outdoor, or atmospheric, aerosols in addition to being principal components of indoor aerosols. Desiccated non-viable fragments of microbial organisms are also common. These fragments have been found in the sub-micron size range. Studies reported that a sizable fraction of both coarse and fine PM in both indoor and outdoor samples was of biological origin [13]. The biological component can be identified specifically by type or component (end toxins), or collectively accounted for in the measurement of protein concentrations (primarily made up of whole and fragmented mold spores, bacteria, and pollen).

The occurrence of Primary biological aerosol particles (PBAP) in the atmosphere has been reported by Jaenicke (2005). Numbers found for airborne bacteria are typically smaller than for spores. Due to their vastly smaller mass. Their contribution to the total aerosol mass becomes negligible. The same is the case for viruses, which are not considered to occur as discrete particles but instead to form clusters or droplets.

We will focus our evaluation on the mass fraction of Primary biological aerosol particles (PBAP) to PM10 concentration, in order to better understand their contribution to particulate air pollution. We will not consider any other potential health related issues (toxic or pathogenic properties), which are more commonly the basis for considering bioaerosols. Moreover, we will not discuss particle number concentrations which are essential for the Primary biological aerosol particles (PBAP) role.

The topic of this paper represents an important research area for the biological significance and removal of fine particles in air. The aim of this work is to assess quantitatively the capability of trees in removing airborne soot particles of submicron and ultra-fine sizes. The work in this paper is only beginning and rough. One exception to this that we do not study the toxicology and the short term/long term effect of these particles to biological, and plan to take it up as a future work.

\section{Materials and Methods}

We have conducted experiments on deposition particles of leaves. Particle size distribution spectrum and main heavy metal components of the particles had been tested. This study collected four kinds of leaf samples in three locations of the tree. Leafs were equilibrated and weighed for comparison with washed leafs for recording mass gain. The unused leaves were stored in plastic petri dishes until needed. A minimum of 20 numbers of leaves were prepared as samples. Four kinds of trees were chosen such as Nerium oleander, Pittosporum tobira, Osmanthus fragrans, Japan Aucuba. Figure. 1 show pictures of the tested leaves according to tree species.

For other tree species, every leaf used for the experiments was scanned using an image scanner and the scanned image was transformed into the black and white image, i.e. the scanned image of the leaf appeared black on the white background. Then, the surface area of each tree leaf was produced by the Adobe Photoshop CS2 program. By calculating the area of the black image. When 
some images of known areas printed on A-4 papers were scanned and then the total areas of those images were obtained using the Adobe Photoshop CS2 program, the error of this scanning method was expected to be within $0.5 \%$. It should be noted that the surface area obtained in this way was a kind of projected area, i.e. the veins and other structures on the leaves were not considered in determining the surface area.

The amount of particles deposited and the average single leaf and deposition amount of per unit area in four kinds of leaves are shown in table 1. It can be known from Table 1that Nerium oleander has biggest ability of capturing particles for these four kinds leafs. Why it has this ability? In order to understand collection mechanisms of Nerium oleander, we make the SEM image to Nerium oleander leaves and analyze it.

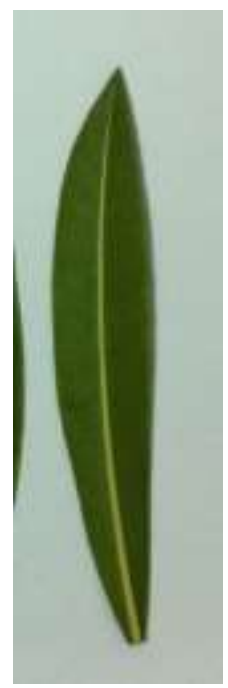

(1) Nerium oleander

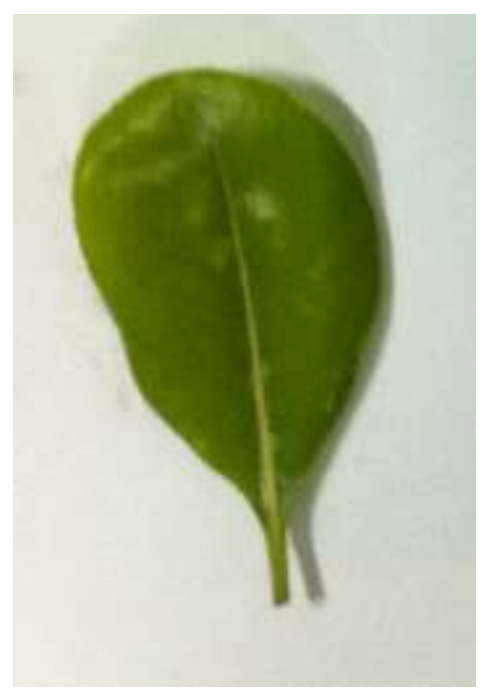

(2) Pittosporum tobira

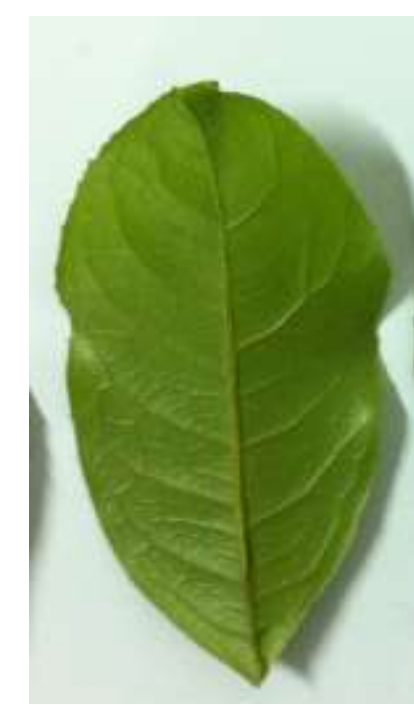

(3) Osmanthus fragrans

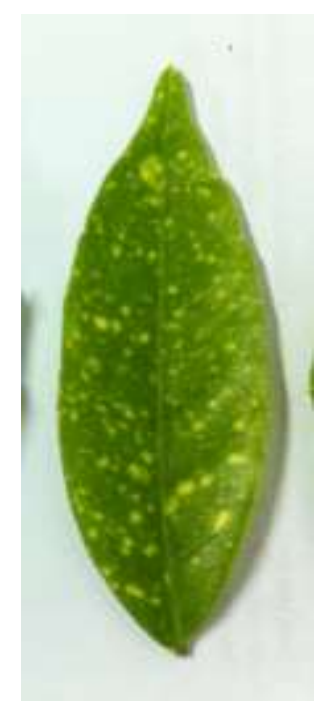

(4) Japan Aucuba

Fig. 1. photo of four kinds of tree leafs

Table 1. The amount deposited in leaves

\begin{tabular}{|c|c|c|c|}
\hline plant & $\begin{array}{c}\text { The average single leaf area } \\
\text { (square centimeter) }\end{array}$ & $\begin{array}{c}\text { The total amount } \\
\text { deposited in leaves }(\mathrm{g})\end{array}$ & $\begin{array}{c}\text { Deposition amount per } \\
\text { unit area }\left(\mathrm{g} / \mathrm{m}^{2}\right)\end{array}$ \\
\hline $\begin{array}{c}\text { Nerium oleander } \\
\begin{array}{c}\text { Pittosporum } \\
\text { tobira }\end{array}\end{array}$ & 16.15974068 & 0.4135 & 12.79414096 \\
\hline $\begin{array}{c}\text { Osmanthus } \\
\text { fragrans }\end{array}$ & 9.400350182 & 0.176 & 9.361353385 \\
\hline $\begin{array}{c}\text { Japan } \\
\text { Aucuba }\end{array}$ & 44.69807549 & 0.483 & 5.40291718 \\
\hline
\end{tabular}

After samples were collected, each leafs was placed in a separate petri dish, the dish was labeled and the date and weight of sampled were recorded. Leaf gravimetric change was recorded. After weighing, each leaf was placed back into the petri dish, and put back into the environmental chamber until analysis. 
At the time of analysis, the leaf was suspended in sterile, phylogenic-free, glucan-free water and analyzed for total protein. Each leaf sample was placed into a separate sterile container with 30 mole buffered detergent, and shaken vigorously for $30 \mathrm{~min}$ to elute the collected PM into solution.

We used a thousand electron microscope magnifications to observe the morphology of the particle or bio-particle firstly, then selected some specimen and send them to be making a large photo via using scanning electron microscope (SEM). Particle existing in tree leaves was shown in Fig. 2. It shown that tree leaves can collect fine particles. In those particles included some bio-particles. It will be discussed in section 3.2.

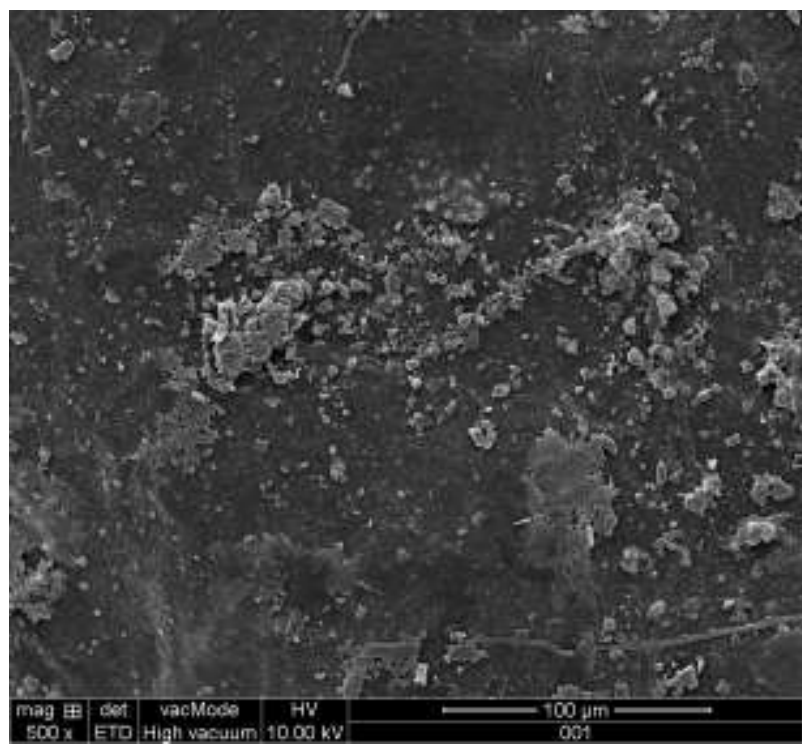

(a)

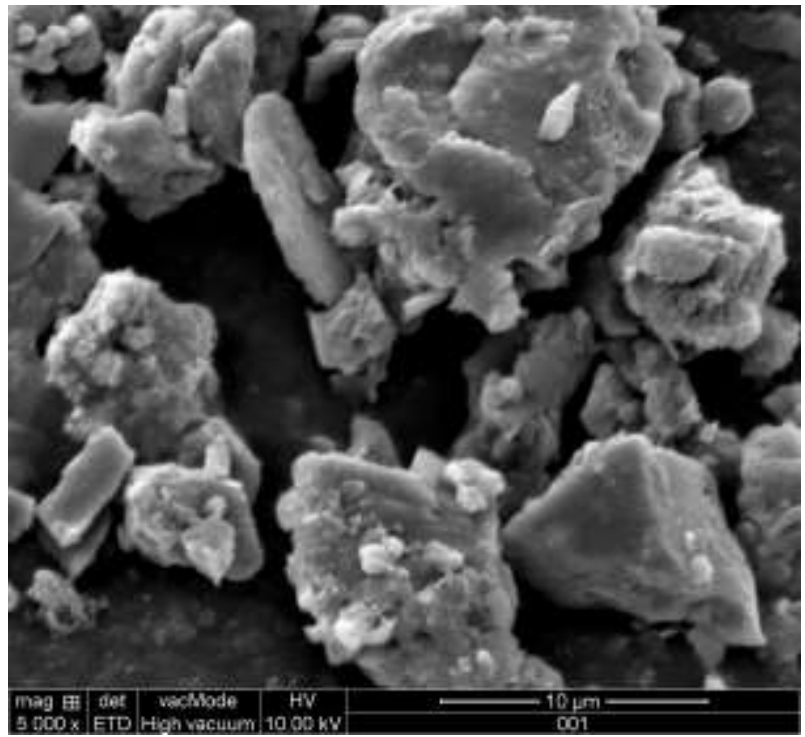

(b)

Fig. 2. An example of particles existing in tree leaves

\section{Results and Discussion}

Particles Size Distributions. For sampling leave an elongate rod and a sealed plastic bag to be used. The coarse particles ( $r>2 \mathrm{um}$ ) were sampled on colored glycerin jelly (Matthias, 1987). Biological particles got stained and could be distinguished in opposite to the non-dyed particles using a light microscope.

The small particles $(0.2 \mathrm{um}<\mathrm{r}<2 \mathrm{um})$ were examined in a scanning-electron-microscope (SEM) equipped with an energy dispersive X-ray spectrometer (EDX) after sampling on graphitic foils. The morphology of particles was utilized to characterize. The literature and own experiments showed, that the biological particles have special morphology (spheres, rods, characteristic forms) together with a special elemental composition. Based on scanning-electron-microscope (SEM) analysis particles of leafs were dividing into biological or non-biological particles.

Fig.3 showed SEM image of Nerium oleander collected particles. It can be seen that most particles were collected by surface roughness of tree leaves. Leaf veins and texture play an important role in collecting fine particles in the tree leaves. This study shows that the removal of airborne particles of submicron and ultra-fine sizes could be influenced by the surface roughness of tree leaves, i.e. the veins and other structures on the leaves. For example, nerium oleander has many veins and complex texture so that it had a bigger capability of collecting fine particles. 
The leaf samples were tested by using the Nan particles measures soft and the size distributions of the biological and non-biological aerosols were obtained. As for non-biological aerosols, their size distribution showed a single-peak distribution, but the particle size of the highest proportion was different in different magnification SEM image. As a result, we found that the distribution of the particles in the leaves was Poisson distribution, 0-1 micro-meter particles is about $5 \%-8 \%$ of total aerosol particles. The particles diameter was about smaller than 100um. Up to $40 \%$ of the total aerosol particles of a size were about 1-3 micro-meter in diameter.

In the submicron range, we did not find such a strong distribution variation for two same magnification SEM images. Furthermore, we found particles size distribution strong dependence on the image magnification which has relation to the numbers of larger particles and tiny particles.

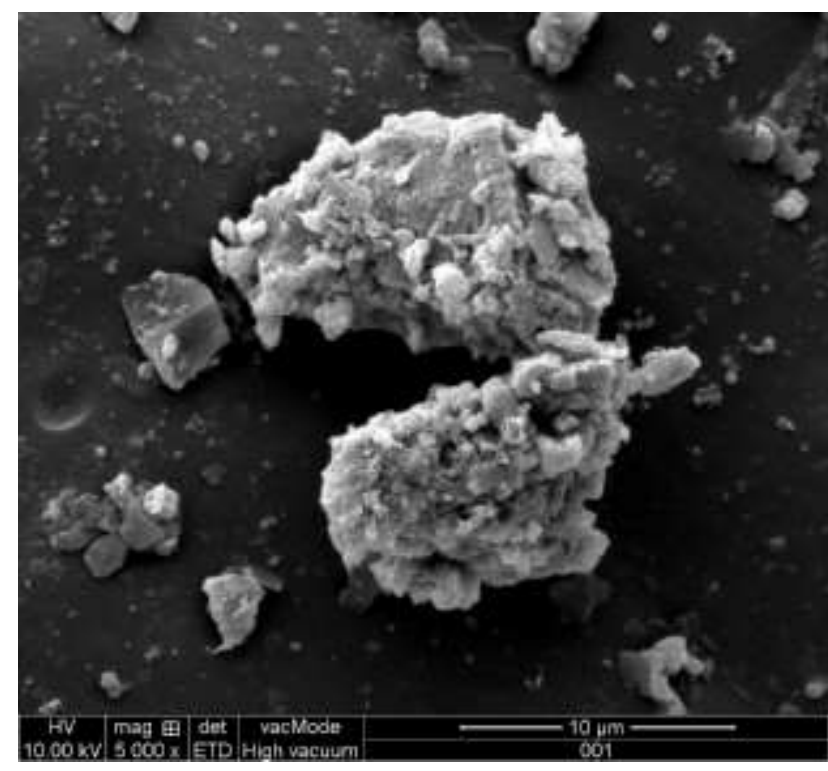

(a) High-magnification image(5000x)

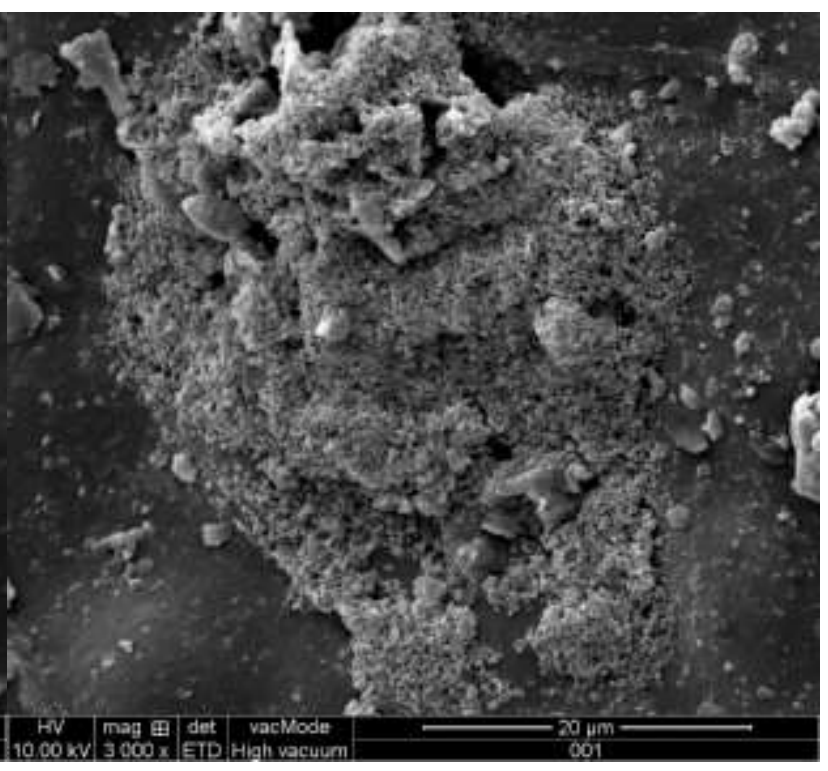

(b) High-magnification image(3000x)

Fig. 3. SEM image of Nerium oleander leaf collected particles.

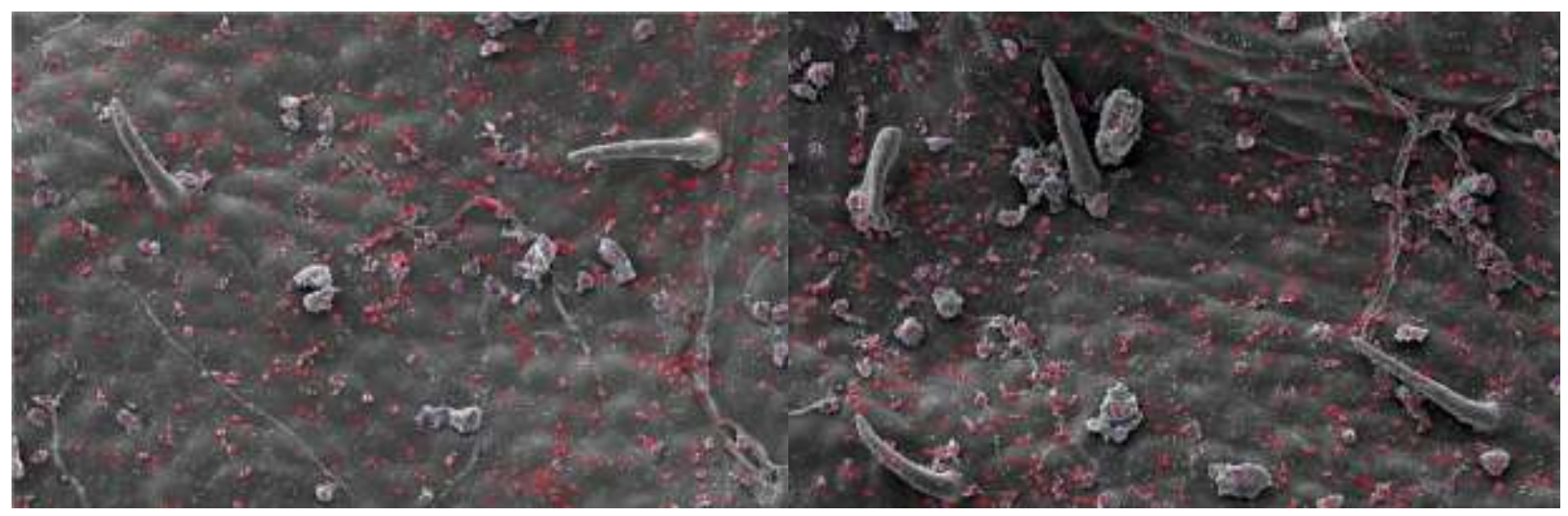

(a)Total particles is 581 , magnification is 500

(b) total particles is 562, magnification is 500

Fig. 4. Aerosol deposits collected on the Nerium oleander leaf of two same magnification SEM photograph

Hyphae, Conidia and Spores of Fungi. Microbes play an important role for green plants which includes promoting health, regulation and protection, if there is the Microbes be observation in plants and how they spread to atmospheres environmental is microbial research topic in the future 
work for us, there are some literature conducted a similar study. Another possible direction for future work can be to analyze detailed microbes components of the particles in the leaves.

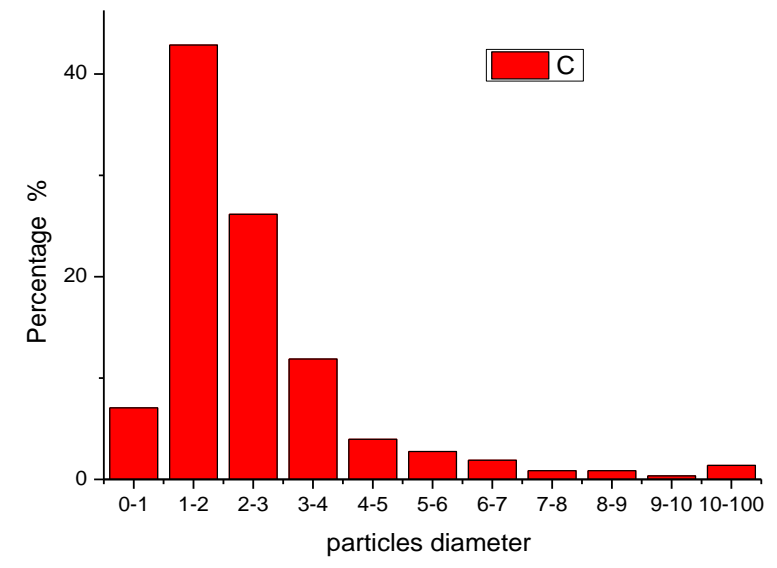

(a)first image

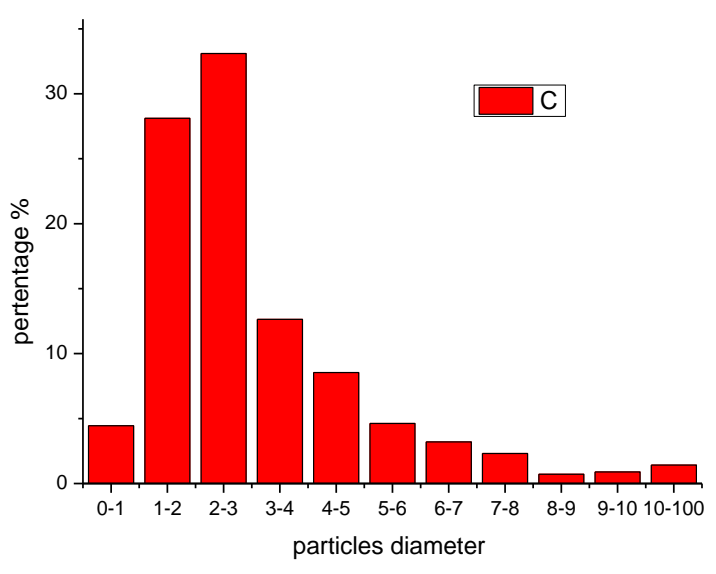

(b) second image

Fig. 5. particles distribution of nerium oleander leafs

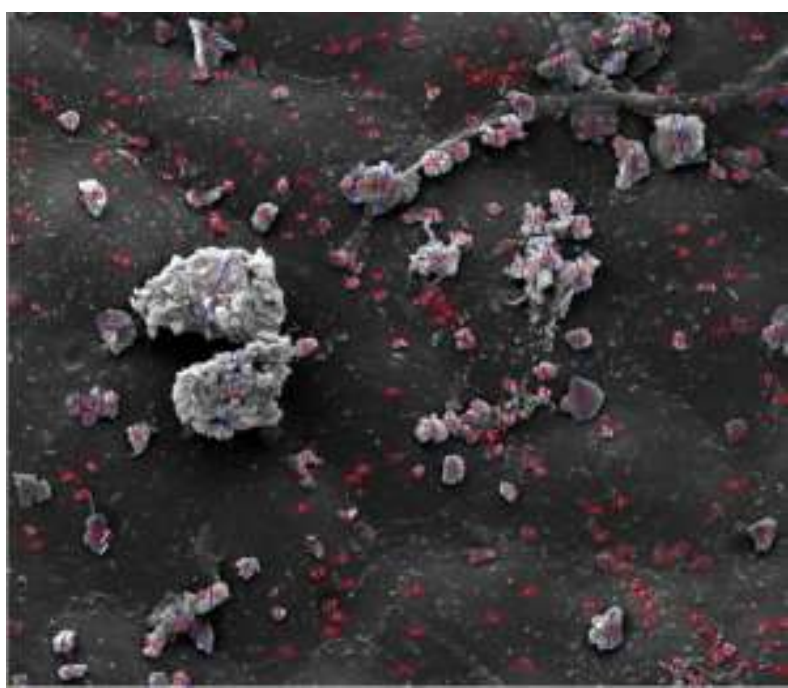

(a) Total particles is 230 , magnification is 2000

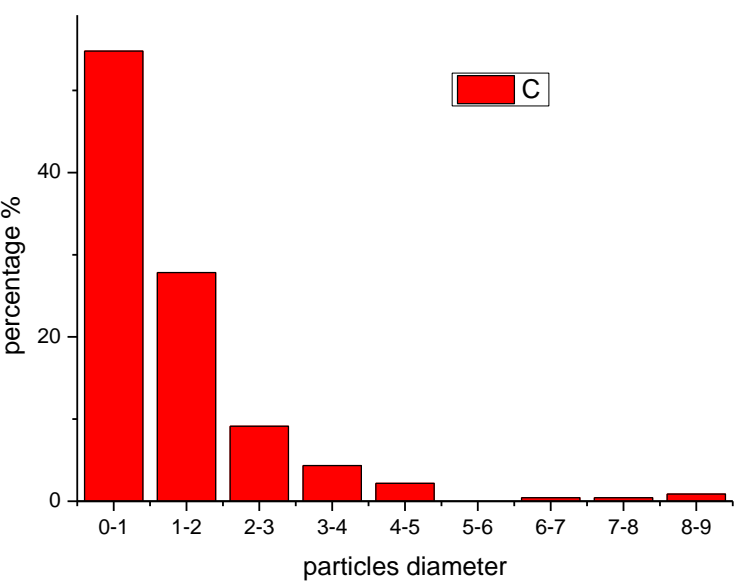

(b) particles distribution in image

Fig. 6. Aerosol deposits collected on the Nerium oleander of high magnification SEM image.

Green plants outdoors should be considered potential allergen sources and tested component of particles in different leaves; particles of the leaf contain many bio-aerosol particles. Hyphae, conidia and spores of fungi may be existing in those particles. We observed morphology of particles to find some bio-particles existing in tree leaves, an example leafs of bio-particles existing in tree leaves was shown in Figure 7. The results were same literature, more morphology of bio-particles was shown in Figure 8. In Figure. 7 (a) consists of two fragments of hyphae. Figures. 8 shows a series of fungi products, all of which appearing to be conidia or spores[14].

Particulate Matter Composition and Toxicological Analysis. Heavy metal content determined by acid digestion measurements, firstly set leaves on a hot plate to heat digestion dust filter, and then, using the full spectrum of plasma emission spectrometer to direct reading concentrations of heavy 
metals. Samples were analysed for heavy metal content, including $\mathrm{Cd}, \mathrm{Co}, \mathrm{Cr}, \mathrm{Cu}, \mathrm{Ni}, \mathrm{Pb}$ and $\mathrm{Zn}$. Its main ingredients are listed as: $\mathrm{Zn}=38.2 \% ; \mathrm{Cu}=10.1 \% ; \mathrm{Pb}=4.4 \% .>\mathrm{Ni}>\mathrm{Co}>\mathrm{Cr}$
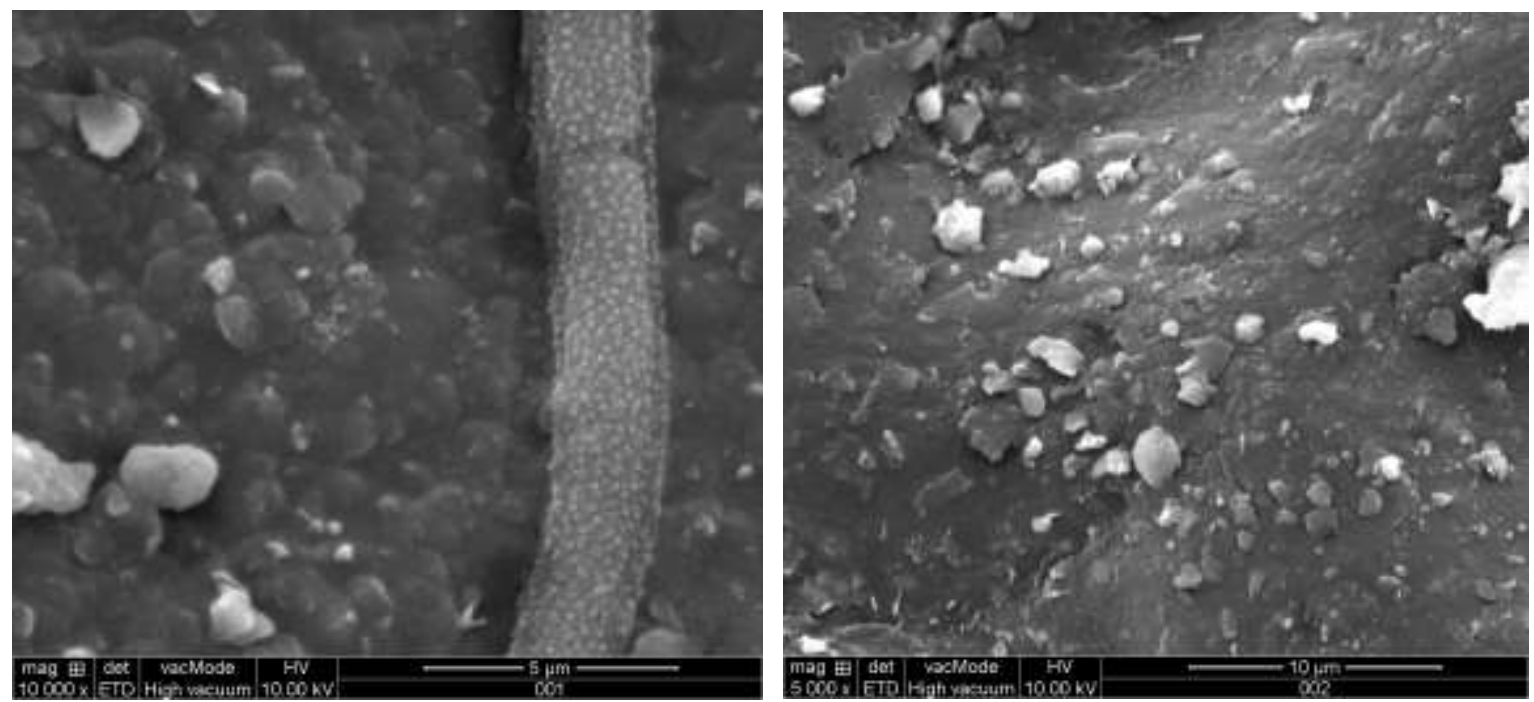

Fig. 7. a example leafsof bio-particles existing in tree leaves
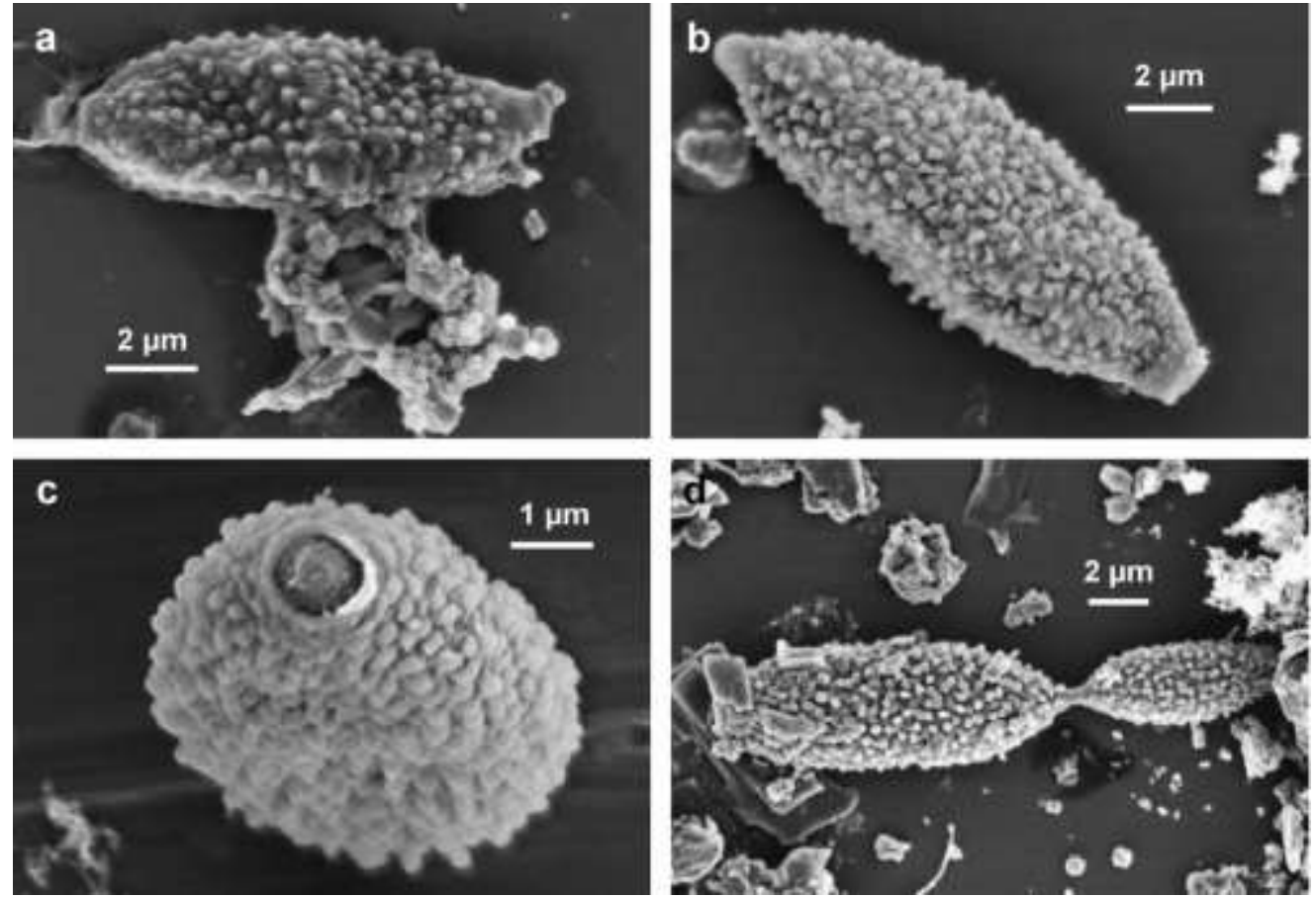

Fig. 8. (A) - (d) Different examples of conidia of fungi species C. herbarium.

Its ingredients in descending order, they are sorted as follows: $\mathrm{Zn}>\mathrm{Cu}>\mathrm{Pb}>\mathrm{Cd}$.

Leaves particles matter was significantly associated with heavy metals. It shows that heavy metals of leaves were come mainly from the soil and leaves are affected directly by deposition of atmospheric pollutants.

It can been seen from SEM observe that deposition particles may interference plants absorb 
sunlight and carbon dioxide as well as the process of release oxygen and waters, thus deposition particles in leaves may affecting the health and growth of plants, more effects on leaves function will be detail studied in next work.

Laden et al [16] studied the effects of particulate matter component on mortality variation. The results showed that particulate matter component come from the transportation emissions impact on mortality is greater than that of the particulate matter component come from coal-derived particles. Such as motor vehicles, coal-fired emissions particles concentration of PM2.5 increased per $10 \mu \mathrm{g} \bullet$ $\mathrm{M}-3$, respectively, daily mortality increased $3.4 \%$ and $1.1 \%$.

Laden et al [16] showed that PM2.5 exposure may lead to pathological and physiological changes in the blood system, including a series of inflammation and coagulation and heart rate, and thus constitute a risk factor for cardiovascular system.

It can been known from Table 1 that average deposition amount per unit leaf area was $7.554 \mathrm{~g} / \mathrm{m} 2$, leaf area index was usually $7-15$, if we selected average leaf area index value was 11,we can estimates that an acre of forest can absorb 20 tons of dust in one years. It shows that the tree leaves can reduce the concentration of particulate matter in the air, and reduce the daily mortality of a population.

\section{Conclusions}

This study monitored and compared four kinds of leaves in an urban site of Shanghai. China, particles distribution was analyzed; understanding the concentrations and distribution of airborne aerosol can assist in our ability to cope with the reality of global warming. While measures are taken in order to reduce reduce and reverse the debilitating environmental effects of global warming, monitoring airborne aerosol can help improve public health awareness and serve as a spatial indicator of climate change.

A comprehensive monitoring study of particles removing of leafs would contribute to the understanding of airborne aerosol control. Little knows about the generation process of bio aerosol. Without information on the generation process, it is difficult to assign the responsibility of an emitter. While bio aerosol is definitely the result of biological processes, it is not at all clear whether such processes should be considered natural. From atmospheric concentrations alone, without a proper source term, it is virtually impossible to correctly attribute the origin of bio aerosol in the atmosphere. Consequently, an exemption from a requirement to reduce the concentrations, being a natural source, cannot be substantiated. From the perspective of the citizen's health, there is no reason for a differentiation between this source being natural or anthropogenic: as stated above, no information is available that this source, if at all natural, is less hazardous or to the contrary, atmospheric concentrations observed from natural sources should rather lead to enhanced abatement of man-made PM emissions in order to arrive at a balanced situation of acceptable air quality.

Four kinds of leaf samples were analyzed for mass; particles distribution was analyzed in the SEM image. The results indicated that between 5\% and 8\% PM mass concentrations were made of ambient bioaerosols. Experimental test was conducted repeatedly. The distribution of the particles in the leaves was Poisson distribution. The distribution of aerosols documented in this study can serve to fully understand exposure to airborne aerosol control. This can be significant to protect atmospheric environmental. Another possible direction for future work can be to examine whether 
or not the particles matter of leaf in the tree canopy can generate the spread of germs and detailed components of the particles, the toxicology study and the short term/long term effect of these particles in biology. The data presented in this paper depict the mass and particles distribution of tree leaves. That mass of particles is a direct measurement of the portion of total PM. This article provides an investigation of leaf containing bioaerosol concentrations and particles distribution in urban of Shanghai, China. Additional studies are required to further characterize plant collecting particles mechanism, as well as the role they play in affecting air environmental variation.

\section{Acknowledgment}

This paper received the support of National natural science foundation of China Project 51178094 and 41371445 . We wish to thank the anonymous referees for their detailed comments and insightful suggestions, which helped in our work.

\section{References}

[1] Baldauf, R, Thomas, E, \& Isakov. Traffic and meteorological impacts on near road air quality: summary of methods and trends from the Raleigh near road study. Journal of air and waste management. 2010, 58 (7), 865.

[2]Breton, M.C, Garneau, M, Fortier, I, Guay, F, Louis, J. Relationship between climate, pollen concentrations of Ambrosia and medical consultations for allergic rhinitis in Montreal, 1994Y2002. Science of the Total Environment, 2006,370, 39-50.

[3]Moorcroft, P.R, Pacala, S.W., Lewis, M.A., Potential role of natural enemies during tree range expansions following climate change. Journal of Theoretical Biology, 2006,241, 601-616.

[4]Schneider, D., Bernard, B., Defile, C., Gehrig, R., Effect of climatic changes on the phonology of plants and the presence of pollen in the air in Switzerland. Journal of Allergy and Immunology (Paris), 2002, 34, 113-116.

[5]Peteet, D., Sensitivity and rapidity of vegetation response to abrupt climate change. Proceedings of the National Academy of Science United States of America, 2000, 97, 1359-1361.

[6]Pawankar, R., Baena-Cagnani, ea. tl, State of world allergy report 2008: allergy and chronic respiratory disease. Journal of world allergy organization. 2008, S4-S17.

[7] Samet, J.M., Spengler, J.D., 1991. Indoor Air Pollution - a Health Perspective. Johns Hopkins University Press, Baltimore, MD.

[8]Menetrez, M.Y, Foard K., Ensor, D.S, An analytical method for the measurement of nonviable bio-aerosols. Journal of the Air and Waste Management Association, 2001, 51, 1436-1442.

[9]Donaldson, K., MacNee, W., Potential mechanism of adverse pulmonary and cardiovascular effects of particulate air pollution (PM10). International Journal of Hygiene and Environmental Health, 2001,203 (5-6), $411-415$.

[10]Douwes, J, Thorne, P, Pearce, N, Hedrick D. Bio-aerosol health effects assessment progress and prospects. Annals of Occupational Hygiene, 2003, 47 (3), 187-200.

[11]Gilmour, M.I., Jaakkola, M.S., London, S.J., Nel, A.E., Rogers, C.A., 2006. How exposure to environmental tobacco smoke, outdoor air pollutants, and increased pollen burdens influences the incidence of asthma. Environmental Health Perspectives114, 627-633.

[12]Ghio, A.J., Kim, C., Devlin, R.B., 2000. Concentrated ambient air particles induce mild pulmonary inflammation in healthy human volunteers. American Journal of Respiratory Critical Care Medicine 162 (3, Pt 1), 981-988.

[13]Husman, T., 1996. Health effects of indoor-air microorganisms. Scandinavian Journal of Work, Environment \& Health 22, 5-13.

[14]Wan, G.H., Li, G.S., 1999. Indoor end toxin and glucan in association with airway inflammation and systemic symptoms. Archives of Environmental Health 54(3), 172-179.

[15]Schwartz, D.A., Thorne, P.S., Yagla, S.J., Burnmeister, L.F., Denchuck, S.A., Watt, J.L. The role of end toxin in grain dust induced lung disease. American Journal of Respiratory Critical Care Medicine $1995.152(2), 503-600$.

[16]Targonski, P., Persky, V., Rameskrishnan, V., 1995. Effect of environmental molds on risk of death from asthma during the pollen season. Journal of Allergy and Clinical Immunology 95, 955-961. 
[17]Menetrez, M.Y., Foarde, K.K., Webber, T.D., Dean, T.R., Betancourt, D.A., 2007a. An evaluation of the protein mass of particulate matter. Atmospheric Environment, 41, 8264-8274.

[18]Monn, C., 2001. Exposure assessment of air pollutants: a review on spatial heterogeneity and indoor/outdoor/personal exposure to suspended particulate matter, nitrogen dioxide and ozone. Atmospheric Environment 35, 1-32.

[19] K. Wittmaacka, H. Wehnesb, U. Heinzmannb, R. Agerer. An overview on bio-aerosols viewed by scanning electron microscopy, Science of the Total Environment 346 (2005) 244-255

[20] Laden F,Neas L M,Dockery D W,et al. Association of fine particulate matter from different sources with daily mortality in six U. S. cities. Environmental Health Perspectives, 2000,108 (10) : 941 - 947. 